\title{
Behaviors of Carriers in Doped Polymer LEDs
}

\author{
Chung-chih Wu \\ Graduate Institute of Electro-optical Engineering and department of Electrical Engineering \\ National Taiwan University, Taipei, Taiwan \\ chungwu@cc.ee.ntu.edu.tw
}

In this work we describe the characteristics of single-organic-layer LEDs based on doped polymers. Behaviors of carriers are correlated to material properties and are examined with various carrier transport models.

Organic LEDs, Doped polymer LEDs, photoluminescence 


\section{Introduction}

Organic light emitting diodes (OLEDs) are attractive for flat panel display applications because of ease and potential low cost of fabrication, the lack of a requirement for crystalline substrates, the versatility in colors, and demonstrated performance.

Among various materials for OLEDs, doped polymers are distinct by the convenience in color and electrical tuning with dopants. In this paper, by correlating device characteristics with material properties and various carrier transport models, we seek to understand the behaviors of carriers inside this class of materials and devices.

\section{Doped Polymer Films and Devices}

Doped polymers under investigation are based on the polymer poly(N-vinylcarbazole) (PVK) which is a relatively good hole transport polymer but is only a weak emitter of light in the deep blue (emission peak $\sim 430 \mathrm{~nm}$ ). Adding dyes into the PVK increases both photoluminescence (PL) and electroluminescence (EL) efficiencies because of the high radiative probabilities of the dyes and because excitons become localized on the dyes and cannot migrate to non-radiative centers. Fig. 1 shows the spectra and Fig. 2 the integrated PL intensity for 340 $\mathrm{nm}$ excitation as a function of the content of $\mathrm{Bu}-$ PPyV in PVK. Bu-PPyV is a fluorescent lowmolecular-weight polymer that emits in green at low concentrations and in the red at high concentrations (due to excimers [1]). Qualitatively similar PL results are obtained for either two-component (PVK/Bu-PpyV) or three-component (PVK/PBD/BuPpyV) blends as shown in Fig. 1, Fig. 2 and Fig. 3. (PBD is a well-known small molecule electron transport agent that was added to the blends in solution before spin-coating [2].) Note efficient energy transfer is achieved between the PVK, where most absorption occurs, and the dye, so that at 1 weight $\%$ dye concentration almost no emission from the PVK can be seen.

Devices were fabricated from these blends by depositing the films by spin-coating on ITO-coated glass substrates, followed by evaporated metal contacts. EL spectra for different dye content show the similar gradual trend of the PL as the dye content is increased. In devices without an electron transport agent, the maximum EL efficiency was $0.3 \%$ (Fig. 2). By incorporating PBD to help transport electrons into these "materials, the qualitative results were the same as a function of dye content, but the maximum efficiency was increased to $0.8 \%$ (Fig. 3, Fig.4) and the operating voltage were decreased as shown in Fig. 5.

\section{Discussions}

Note from Fig. 2 and Fig. 3 that the maximum relative increase in PL efficiency in the blends is only about two, while the EL efficiency increases by about a factor of 10 as the dye is added. This leads us to conclude that excitons are formed on the dye under electrical operation by the sequential capture of electrons and holes, rather than exciton formation in the host followed by transfer to the dye [3]. The energy levels (HOMO's and LUMO's) of these materials determined from the electrochemical technique are consistent with this picture [3].

In the optimized doped polymer OLEDs, the reasonable EL efficiency suggests of comparable injection abilities for both holes and electrons. It is therefore in general a double-carrier injection situation. In general, the analysis would be complicated by how to describe the injection process from the electrode, the transport process in the bulk, and the recombination process. As an initial effort to understand the behavior of carriers in these doped polymer OLEDs, the simpler case of single-carrier injection devices was analyzed first. 
Fig. 6 shows the I-V characteristics of ITO/PVK/Mg:Ag/Ag device. Considering its relatively low EL efficiency $(<0.01 \%)$ and the much lower mobility of electrons than that of holes, the I-V characteristics of this device are mainly governed by holes and can be seen as a single-carrier device. In this I-V curve, the current strongly increases for voltages above a critical voltage. This type of behavior resembles the space charge limited current (SCLC) occurred in a low mobility material with an exponential distribution of trap-level energies within the energy gap $[4,5]$ :

$$
J=N_{\vee} q \mu_{p}\left(\frac{\varepsilon}{q N_{t}}\right)^{m} \frac{V^{m+1}}{L^{2 m+1}} C(m)
$$

with $J$ the current density, $N_{t}$ the total density of traps, $k T_{t}$ the energy characterizing the trap distribution, $m=T_{t} / T, \varepsilon$ the permitivity of the organic layer, $N_{v}$ the effective density of states in the valence (HOMO) band, $\mu_{p}$ the mobility of holes, $L$ the thickness of the organic layer, and $C(m)=m^{m}(2 m+1)^{m+1}(m+1)^{-}$ $(2 m+1)$.

It should be noted from (1) that the trap distribution parameter $T_{t}$ not only determines the slope of the $\log I-\log V$ plot, but also the thickness $(L)$ dependence in the trap-charge-limited regime. According to (1), $V$ is approximately proportional to $L^{2}$ at a constant current if $m \gg>1$. This is examined with devices of different PVK thickness and is shown in Fig. 7. It is seen that the linear fits of $V-L^{2}$ data intersect the $V$-axis at voltages comparable to the total drive voltage. This is not consistent with the TCL SCLC description, which predicts nearly zero intersection voltage. It should be noted that the validity of (1) is based on the assumption that the contact is rather "ohmic". That is, the voltage across the contact is small compared to the voltage across the bulk and the boundary condition for the electric field distribution at the injection contact is small compared to the maximum field in the bulk $[4,5]$. From these examinations, we therefore conclude that the ITO/PVK is not ohmic and that the injection process also has significant contribution to the $\mathrm{I}-\mathrm{V}$ characteristics.

Establishing the contacts are a major source of the voltage drop in the PVK device, we now examine two models of carrier injection, Fowler-Nordheim (F$\mathrm{N})$ tunneling and thermionic emission, to see if they can account for the observed voltage drop in our devices. Assuming the minor space-charge effect and a triangular barrier at the electrode-organic interface under an applied field $F$, the F-N theory predicts [6]:

$$
J=\left(\frac{q^{3}}{8 \pi h}\right) \frac{F^{2}}{\phi} \exp \left[\left(\frac{8 \pi \sqrt{2 m^{*}}}{3 q h}\right) \frac{-\phi^{3 / 2}}{F}\right]
$$

with $h$ the Planck's constant, $\phi$ the barrier height, and $m^{*}$ the effective mass of the charge carrier. In general, the device I-V only follows the F-N relation over a very limited (high) voltage range in the $\mathrm{F}-\mathrm{N}$ plot $\left(\log \left(U / F^{2}\right)\right.$ vs. $\left.1 / F\right)$. With the effective barrier height extracted from this range, the I-V predicted by (2) exceeds the measured one by many orders of magnitude (Fig. 6). On the other hand, the diffusion-limited thermionic emission theory predicts:

$$
J=q N_{\nu} \mu_{p} F \exp \left[\frac{-q}{k T}\left(\phi-\sqrt{\frac{q F}{4 \pi \varepsilon}}\right)\right]
$$

Again the I-V predicted by (3) is plotted in Fig. 6, where $\mu_{p}=2 \times 10^{-6} \mathrm{~cm}^{2} / \mathrm{V} \cdot \mathrm{s}, \phi=0.4 \mathrm{eV}, \varepsilon=3 \varepsilon_{0}$ and $N_{v}=2.5 \times 10^{19} \mathrm{~cm}^{-3}$ are used. It does not bear much resemblance to the measured one.

From the above examination, we are led to the conclusion that in our devices both the bulk transport 
and the contact effects contribute significantly to the device voltage because there is no single process giving reasonable description of the device $I-V$ characteristics. It suggests that there is room for further improvement of these devices.

Since both the injection and bulk properties are important in the doped polymer devices, the dependence of EL on the content of electron transport agents (Fig. 4) may result from the combination of two effects: improved electron injection and improved electron transport as electron transport agents are added [2]. Meanwhile, the hole injection and transport properties may be perturbed by the additives [7]. According to the theory of doublecarrier SCLC [4,5], the balanced injection/transport between electrons and holes leads to a larger extent of charge neutralization within the recombination zone (which could be rather broad in a single layer polymer OLED). The outcome is a significant increase of current in comparison with single-carrier SCLC at the same operation voltage. This may explain why the lowest drive voltage occurs in the doped PVK device with the highest efficiency (Fig. 4, Fig. 5). Furthermore, improved electron transport could move the major recombination zone away from the organic/cathode interface, reducing the metal contact quenching effect.

In summary, we have described the characteristics of single-organic-layer LEDs based on the polymer PVK doped with functional agents. Behaviors of carriers have been correlated to material properties and have been examined with various carrier transport models.

The author is indebted to Dr. J.C. Sturm for some of the work performed at Princeton University.

\section{References}

1. J. Tian, C.C. Wu, M.E. Thompson, J.C. Sturm, and R.A. Register, Chem. Mater. 7, 2190 (1995)

2. H. Tokuhisa, M. Era, T. Tsutsui, and S. Saito, Appl. Phys. Lett. 66, 3433 (1995)

3. C.C. Wu, J.C. Sturm, R.A. Register, J. Tian, E.P. Dana, and M.E. Thompson, IEEE Trans. Elec. Dev. 44, 1269 (1997)

4. K.C. Kao and W. Hwang, Electrical Transport in Solids, Pergamon Press Inc., 1981

5. M.A. Lampert and P. Park, Current Injection in Solids, Academic Press, New York, 1970

6. R.H. Fowler and L. Nordheim, Proc. R. Soc. London, Ser. A 119, 173 (1928)

7. J. Mort and D.M. Pai (ed.), Special Polymers for Electronics and Optoelectronics, Chapman \& Hall (1995)

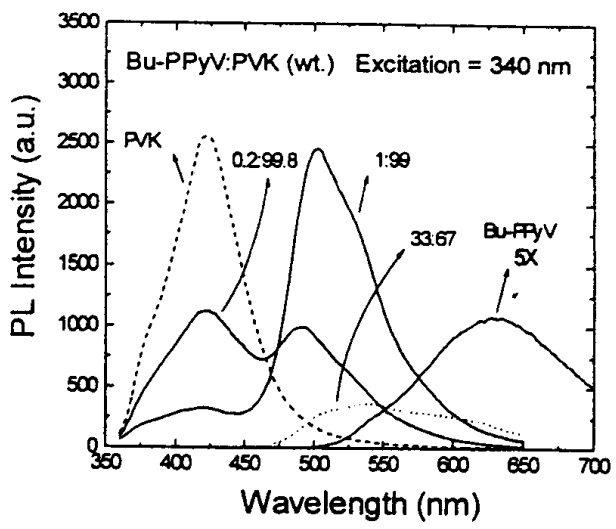

Fig. 1 PL spectra vs. dye content 


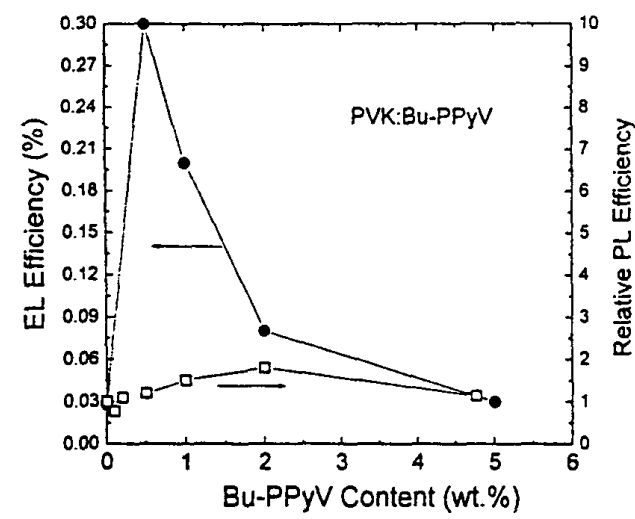

Fig. 2 Emission efficiency vs. dye content

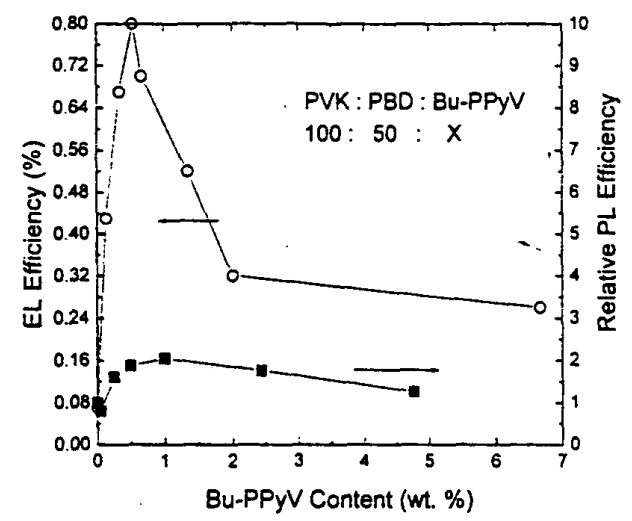

Fig. 3 Emission efficiency vs. dye content

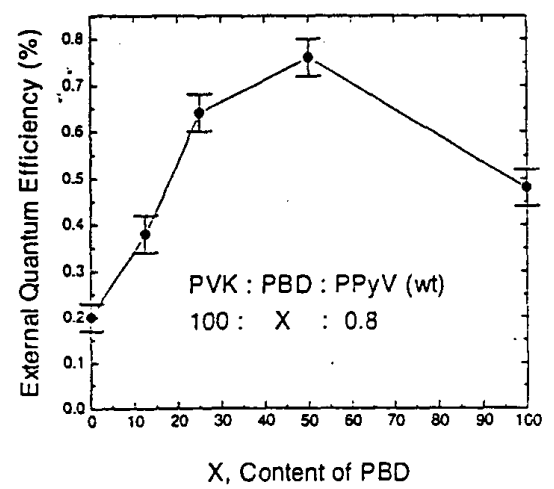

Fig. 4 Emission efficiency vs. dopant content

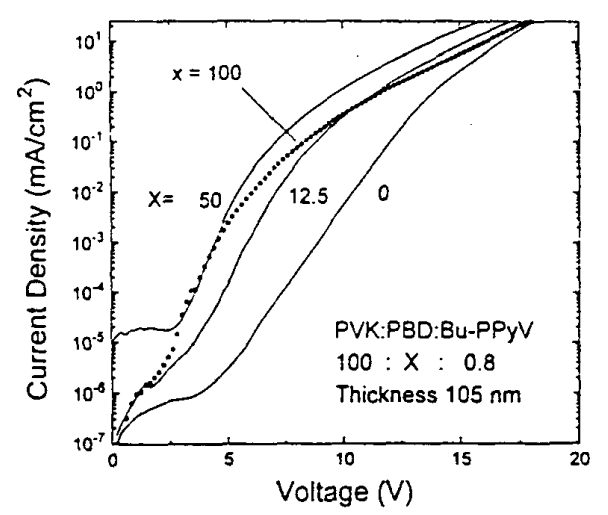

Fig. 5 I-V vs. dopant content

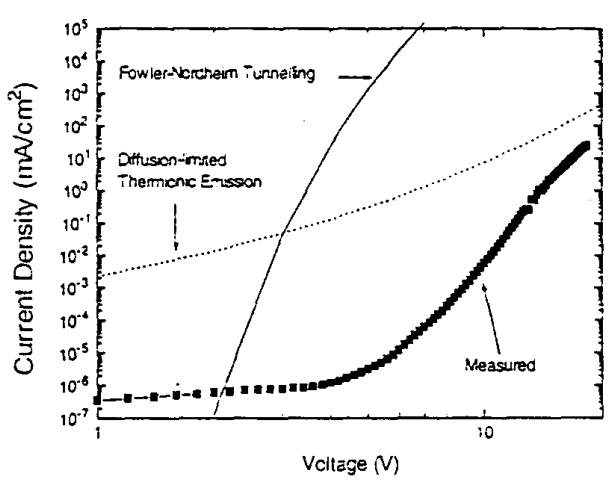

Fig. 6 Measured and calculated I-V

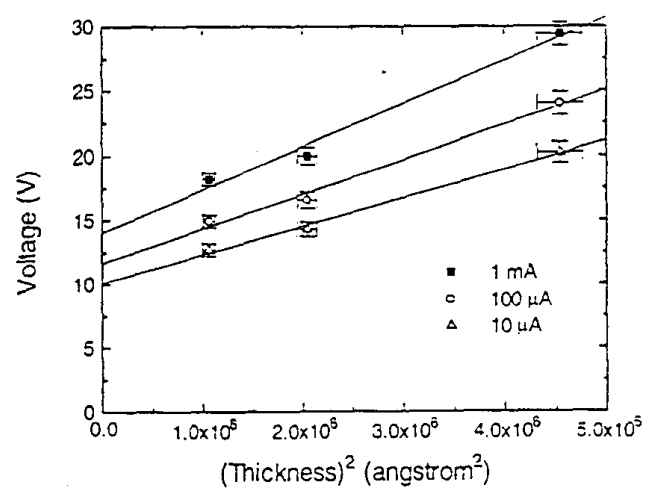

Fig. 7 Voltage vs. (thickness) ${ }^{2}$ 